\title{
Near-Field to Far-Field Transformation on Arbitrary Surfaces via Multi-Level Spherical Wave Expansion
}

\author{
Fernando Rodríguez Varela, Belén Galocha Iragüen, Manuel Sierra Castañer,
}

\begin{abstract}
This paper presents a general technique for the farfield transformation of near-fields measured over arbitrary surfaces and multimode probe correction in an efficient and accurate way. The use of not only one but several spherical wave expansions to model the antenna under test fields allows to incorporate full probe correction for arbitrary orientations with low computation cost. Combining this approach with a hierarchical subdomain decomposition strategy, the transformation problem is solved with a low computational complexity. In this method, the fields produced by neighbor subdomains are gradually aggregated and interpolated following a multilevel scheme, leading to a good scalability with frequency compared with other matrix-based transformation methods. Numerical and experimental results are provided to show the efficiency and capabilities of the proposed algorithm.
\end{abstract}

Index Terms - Antenna measurements, near-field far-field transformation, arbitrary surfaces, spherical wave expansion.

\section{INTRODUCTION}

A NTENNA near-field measurements [1],[2] followed by postprocessing techniques constitute a powerful and more accurate characterization tool due to its versatility and low cost compared to their far-field and compact range counterparts. The price to pay is the necessity of a transformation of the measured near-fields to the radiated far-field using the so-called near-field to far-field transformation techniques. Traditionally, field transformation algorithms have focused in the processing of fields measured over canonical surfaces (planes [3], cylinders [4] and spheres [5] ) using their respective modal expansion techniques, where the fields are expanded in a set of orthogonal basis functions enabling field transformations straightforwardly.

Modal expansion techniques may pose strict requirements regarding the measurement process, since canonical acquisition surfaces are needed to maintain the orthogonality of the basis functions. In addition, in the case of spherical near-field measurements, the most accurate [6], the antennas used as probes are required to be rotationally symmetric in their radiation pattern to obtain a computationally efficient transformation. This fact reduces the flexibility of near-field measurements, since a collection of probes is required to cover a wide range of frequencies.

Also, classical near-field measurement systems must take electromagnetic field samples on a regular grid of points over canonical surfaces. However, in certain applications a higher flexibility in the sampling scheme is desired or even required. That is the case of portable and/or aerial measurement systems [7], [8] where these regular sampling grids cannot be realized, or advanced robotic positioners [9], where arbitrary measurement surfaces can be employed to better adjust the antenna shape or increase the sampling rate in critical areas.

Therefore, it is obvious that alternative near-field to far-field transformation algorithms to the modal expansion techniques are necessary to accommodate versatile near-field measurements for fast antenna characterization. In fact, an extensive work has been developed during the last years to obtain efficient solutions for the transformation of near-fields measured with arbitrary probes and measurement surfaces.

In this sense, several approaches have been presented for the transformation of spherical near-field measurements performed with high-order (arbitrary) probes, depending on the angular scanning [10] and probe modal content [11]-[14]. Nevertheless, these techniques can only be applied for regular spherical measurement surfaces.

Also, irregular sampling has been deeply investigated to reconstruct the far-field from a set of near-field non-redundant samples to minimize the measurement time. Optimal sampling interpolation (OSI) expansions are used to efficiently recover the near-field data over a plane [15] cylinder [16] or sphere [17], required by classical near-field to far-field transformations. In [18], the sparse nature of the antenna under test (AUT) modal content is exploited to reduce the number of measurement samples using compressed-sensing optimization. However, these techniques may be regarded as advanced sampling and interpolation schemes over canonical surfaces rather than actual field transformation algorithms.

Some efforts have been made to extend the modal expansion 
techniques to cases different from canonical surfaces with equispaced/equiangular sampling. In particular, matrix inversion-methods [19]- [22] have been proposed, in which the spherical wave expansion (SWE) of the AUT field is obtained solving a linear system of equations from the knowledge of the measurement samples at arbitrary locations. In [22], additional rotation coefficients are incorporated to the SWE formulation to account for arbitrary orientations of the probe. Once the SWE is obtained, it can be evaluated at the far-field straightforwardly.

A complementary technique is the calculation of equivalent currents over the antenna surface [24]. In this case, a matrix equation system is also solved, but the unknowns are, in a general case, magnetic and electric currents defined in a mesh covering the AUT physical surface. It is seen that any type of equivalent representation can be used to model the AUT fields and solve the field transformation problem. In fact, the use of complex source beams has been proposed recently [25] to address arbitrary measurement surfaces.

Regardless of the benefits of each of these equivalent representations, all explicit matrix inversion approaches share the same limitation, which is the poor scalability with the antenna electrical size. The overall computational complexity, in the case the solution is obtained using explicit matrix operations [19],[20],[22],[24],[25], is $O\left((k a)^{4}\right)$, where $k a$ refers to the antenna electrical size, being $k$ the wavenumber and $a$ the radius of the sphere circumscribing the antenna [5]. If the problem geometry and equivalent representation allow it, fast operators instead of matrix multiplication can be used to reduce the computational complexity. In [23] non-equispaced Fast Fourier Transform and interpolations are used to transform near-fields measured over spherical surfaces with irregular sampling and small radial errors (relative to the measurement radius) obtaining a complexity of $O\left((k a)^{3}\right)$. However, to our knowledge this technique has been only tested over quasi spherical surfaces and its applicability to arbitrary geometries seems not feasible.

Using the principles of the Multi-Level Fast Multipole Method (MLFMM) [26], a fully probe-corrected near-field to far-field transformation was proposed in [27]. It can work both with the AUT currents or its plane wave expansion [28]. Matrix-vector products of the iterative inversion algorithm are replaced by fast operators that compute AUT and probe interactions using disaggregation and anterpolation procedures in a multilevel fashion, reducing the computational complexity to $O\left((k a)^{2} \log (k a)\right)$.

A recently proposed multi-level algorithm employing domain decomposition with spatial interpolation and aggregations has proven to be very efficient for the acceleration of integral equation solvers in acoustic scattering [29], physical optics [30] and antenna diagnosis [31]. Based on these principles, this paper presents a fully probe-corrected near-field to far-field technique based on the decomposition of the AUT domain into a hierarchy of subdomains. Each subdomain is modelled using the spherical wave expansion of the contained sources, whose coefficients are found solving an inverse problem. On each iteration of the inversion algorithm, the fields radiated by each subdomain can be sampled over a coarse grid of points taking advantage of its band-limitation properties [32] and then interpolated to the desired grid. Performing these steps in a multi-level scheme reduces drastically the computational complexity down to $O\left((k a)^{2} \log (k a)\right)$, similar to that of the MLFMM-based algorithm [27]. In fact, the proposed technique can be viewed as the spatial counterpart of MLFMM, based on disaggregation and anterpolation in the spectral domain.

Preliminary results of a simplified version of the algorithm have been reported in [33] showing promising capabilities. In this paper the proposed technique is thoroughly developed and tested with the following organization. Section II presents the Multiple Spherical Wave Expansion (MSWE) and its application in near-field to far-field transformation. In section III a multi-level scheme is derived to improve the scalability of the transformation. Finally, in section IV, the proposed algorithm is tested using analytical and measured data of several antennas.

\section{FIELD TRANSFORMATIONS USING MSWE}

An AUT radiating in free space placed at the origin of a spherical coordinate system is considered. According to Love's Equivalence Principle [34], it is possible to define a set of magnetic and electric currents on a surface enclosing the antenna establishing an equivalent problem. Under these conditions, the fields radiated by the antenna and the equivalent currents are exactly the same outside the enclosing surface. Thus, it is said that these currents define an equivalent representation of the AUT.

In addition, let us introduce another equivalent representation of the fields radiated by the AUT, the spherical wave expansion, in which the radiated fields are expanded in a weighted summation of spherical wave functions:

$$
\vec{E}(r, \theta, \varphi)=\sum_{s=1}^{2} \sum_{n=1}^{N} \sum_{m=-n}^{n} Q_{s m n} \vec{F}_{s m n}^{(3)}(r, \theta, \varphi)
$$

being $(r, \theta, \varphi)$ spherical coordinates $Q_{s m n}$ the spherical wave coefficients (SWC) and $\vec{F}_{s m n}^{(3)}$ the spherical wave basis functions.

The spherical wave expansion is, in fact, a more compact representation of the AUT radiation characteristics. It models the fields using a minimum number of coefficients and unlike the equivalent currents approach, a 3D mesh of the AUT model with basis functions is not required. However, SWE is a spectral representation, thus it does not admit a spatial domain decomposition [29], whose benefits will be latter demonstrated, unlike the equivalent currents approach. In this paper, a hybrid equivalent representation is proposed: The Multiple Spherical Wave Expansion (MSWE). The concept of using several SWE to model an antenna was briefly introduced in [35] as an intermediate step to calculate equivalent infinitesimal dipole representation of antennas. In this paper we generalize this formulation for expansions of arbitrary order and for a different purpose.

\section{A. The MSWE equivalent representation}

In MSWE, the surface enclosing the antenna is divided in a 
set of subdomains. Then, the equivalent currents of each subdomain are replaced by their own spherical wave expansion, with its origin coinciding with the subdomain center. The process is outlined in Fig. 1 where the equivalent currents are first represented over the enclosing surface with the subdomain decomposition applied, and then each subdomain has been replaced by an equivalent spherical wave expansion. This can be interpreted as modelling the AUT as an array of smaller antennas, and then modelling the small antennas by its own SWE. The hybrid approach keeps the versatility of the SWE and allows to apply a domain decomposition which will be crucial to reduce the far-field transformation computational cost and apply probe correction.

Considering an AUT modelled with an equivalent surface divided in $L$ subdomains, the radiated field can be expressed as the aggregation of each of the subdomain's field. If each subdomain is expressed by its own SWE centered in a local coordinate system, the field is given by:

$$
\vec{E}(r, \theta, \varphi)=\sum_{l=1}^{L} \sum_{s=1}^{2} \sum_{n=1}^{N_{l}} \sum_{m=-n}^{n} Q_{s m n}^{l} \vec{F}_{s m n}^{(3)}\left(\vec{r}-\vec{r}_{l}\right)
$$

where $\vec{r}_{l}$ is the origin of each SWE that will be placed at the center of each subdomain, and the SWC have an extra index to identify the subdomain they belong. From now on, these will be referred to as multiple spherical wave coefficients (MSWC).

Each $n$-summation is truncated to a given value of $N_{l}$. This truncation number is related to the subdomain size. The bigger it is, the more variations of field it will produce so more harmonics are involved. There exists a rule of thumb [5]:

$$
N_{l}=\left\lceil k a_{l}\right\rceil+10
$$

where $a_{l}$ is the radius of the smallest sphere circumscribing subdomain $l$, and the brackets indicate the largest integer smaller than or equal to the number inside them. By locating the expansions at the subdomain center, the number of MSWC is kept to the minimum. Naturally, rule (3) is valid for $L=1$, which is the case of a conventional single SWE modelling the complete antenna.

The need of locating the expansions over the antenna geometry is a disadvantage as compared to the classical SWE. However, since the equivalent currents are not required to be placed strictly over the physical surface of the AUT, a Huygens
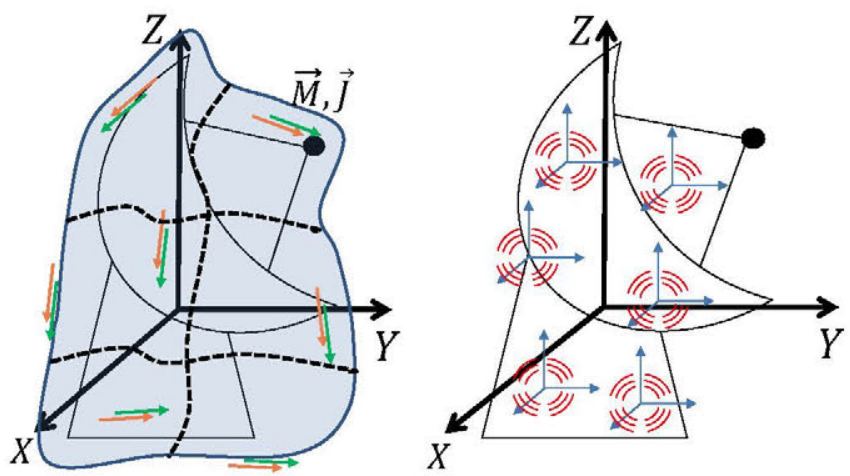

Fig. 1. Schematic view of the equivalent currents over a surface enclosing the AUT with a subdomain decomposition (left) and the modeling of each subdomain by a local spherical wave expansion (right). box (any surface totally enclosing it [34]) can be used. Then, the multiple SWE can be centered over the surface of this box. Therefore, the required a priori knowledge of the antenna to apply this method is the AUT physical dimensions (width, length, height), necessary to know the dimensions of the Huygens box, as compared with the conventional SWE [5] that only requires the minimum sphere radius. It is also noted that the different subdomain's minimum spheres will overlap due to the nature of the discretization. The effect of this overlapping will be an added level of redundancy in the representation, which will increase slightly the number of unknows as compared with the classical SWE.

\section{B. Probe Correction}

Now it is considered that the AUT is being measured by a probe antenna. The probe's output voltage when measuring the field radiated by the transmitting AUT can be computed with Jensen transmission formula [5] that expresses the coupling as a scalar product of AUT and probe coefficients, weighted by some translation and rotation terms. However, this formula only applies to scenarios where the probe is pointing to the coordinate system origin. Therefore, extra rotation terms would be required for an arbitrary case like ours, as shown in [22].

In case AUT and probe are at far-field distance of each other, the transmission equation can be approximated because the probe measures directly the AUT field. It is stressed that both antennas must be at far-field which means that:

$$
d>\frac{1}{2} \frac{\left(a_{A U T}\right)^{2}}{\lambda}, d>\frac{1}{2} \frac{\left(a_{\text {Probe }}\right)^{2}}{\lambda}
$$

being $d$ the distance between AUT and probe, and $a_{A U T}$ and $a_{\text {Probe }}$ their respective sizes as defined previously in terms of circumscribing sphere radius. In most cases the antennas used as probes are electrically small and the second condition is met, being the first the limiting one. With MSWE, subdomain size $a_{l}$ can be made arbitrarily low until condition (4) is met. Then, the measured voltages can be expressed as the sum of the field contributions of all subdomains, weighted by the probe radiation pattern. This procedure is based on the one proposed in [36] to include the probe-distortion effect in equivalent currents methods.

The mathematical foundation is as follows. Consider a subdomain placed at $\vec{r}_{i}$, radiating a field $\vec{E}_{i}(\vec{r})$ (see Fig. 2). The

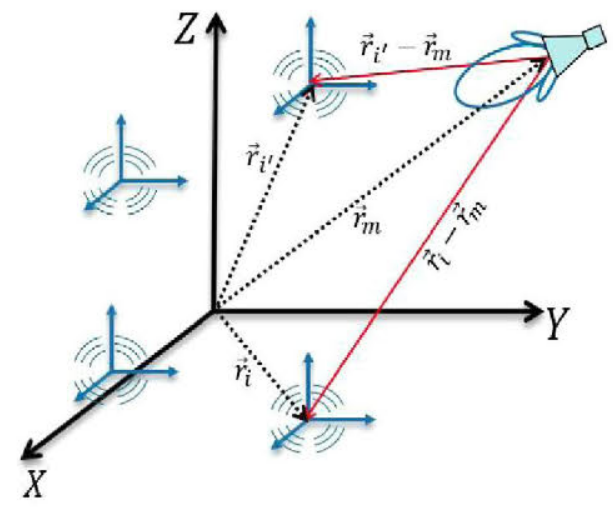

Fig. 2. Illustration of a probe measuring the field of several SWE whose contributions are weighted by its radiation pattern. 
field is measured by a probe with a radiation pattern given by $\vec{f}(\vec{r})$. If both subdomain and probe are at far-field distance of each other, the measured voltage at a point $\vec{r}_{m}$ is given by:

$$
u\left(\vec{r}_{m}\right)=\vec{f}\left(\vec{r}_{i}-\vec{r}_{m}\right) \cdot \vec{E}_{i}\left(\vec{r}_{m}\right)
$$

The field is weighted by the probe radiation pattern in the angular direction corresponding to the subdomain location, as seen from the probe's coordinate system. Fig. 2 depicts a schematic representation of a probe measuring the field radiated by several SWE and the position vectors involved.

Eq. (5) can be extended for a general case when an antenna is composed by $L$ subdomains. In that case, the coupling between the AUT and probe is given by:

$$
u(\vec{r})=\sum_{l=1}^{L} \vec{f}\left(\vec{r}_{l}-\vec{r}\right) \sum_{s=1}^{2} \sum_{n=1}^{N_{l}} \sum_{m=-n}^{n} Q_{s m n}^{l} \vec{F}_{s m n}^{(3)}\left(\vec{r}-\vec{r}_{l}\right)
$$

\section{Near-Field to Far-Field Transformation}

In the previous sections, the MSWE representation of an antenna has been introduced. Closed-form expressions have been given to calculate the radiated field and its coupling with a known probe in terms of the coefficients of this representation. The problem we face now is to find the AUT far-field from the knowledge of a set of near-field samples.

Eq. (6) admits a discrete representation in terms of a matrixvector product:

$$
U=C Q
$$

where $U$ is a vector containing the measured field samples $u(\vec{r})$, $Q$ a vector containing the $\operatorname{MSWC}\left(Q_{s m n}^{L}\right)$ and $C$ a coupling matrix relating both vectors. To obtain the AUT equivalent representation, vector $Q$ must be solved in a least squares sense:

$$
Q=\left(C^{H} C\right)^{-1} C^{H} U
$$

where ${ }^{H}$ denotes Hermitian.

Once the MSWC of the AUT are known, they can be evaluated at far-field using the asymptotic form of the spherical wave functions [5]. This last step is called "direct problem" whereas the matrix inversion is called "inverse problem". Usually, the most challenging part is the inverse problem as it requires a full matrix inversion as compared with the direct problem which is a summation usually implemented by a single matrix multiplication. In practice, an explicit matrix inversion becomes infeasible due to the high number of unknowns involved. Iterative inversion algorithms like Conjugate Gradient (CG) [37] prove to be more efficient as they approach gradually to the solution.

For a proper problem conditioning, $U$ must incorporate enough information about the antenna near-field so that matrix inversion is successful. Measurements with two polarizations over surfaces enclosing the AUT with adequate angular sampling are generally required to solve the least squares problem. The required angular sampling depends on the AUT size. A good estimation is the traditional spherical measurement criterion [5]: $\Delta \theta=\Delta \varphi=\frac{\pi}{N}$, where $N$ is obtained using (3)

\section{Multilevel Spherical Wave AgGregation}

In the previous section, a far-field transformation algorithm has been presented. The basic approach is to model the AUT radiation as a set of multiple spherical wave expansions distributed over a surface enclosing the antenna. An iterative matrix inversion algorithm is started to obtain the coefficients of each SWE and once these are known, the far-field is computed straightforwardly using the asymptotic expression of each SWE and aggregating them.

Most of the processing time is spent in the iterative inversion which consists in repeatedly computing matrix products by $C$. Each matrix-vector product takes $O\left((k a)^{4}\right)$ operations (the number of rows and columns grows quadratically with the antenna electrical size). This is a poor computational complexity that is shared with the rest of explicit matrix-based field transformation techniques [19],[20],[22],[24],[25], and can pose serious limitations in terms of memory and processing time for electrically large antennas.

In this section we seek to reduce this computational cost and to do so we must devise a faster way of calculating matrix interactions, i.e., the field radiated by the MSWC. The Multilevel Spherical Wave Aggregation (MSWA) performs this operation replacing the matrix product with a fast and lowmemory operator. It is stressed that MSWA only constitutes an alternative way of computing a matrix-vector product, the rest of the far-field transformation algorithm remains identical as explained in the previous sections. Therefore, the problem dealt in this section will be just the calculation of the AUT field defined by its MSWE representation, the direct problem.

\section{A. The Bandwidth of Radiated Fields}

When multiplied by a vector $Q$, matrix $C$ can be regarded as an operator that calculates the field of each subdomain at all measurement points $\vec{r}_{m}$ and then aggregates all subdomain contributions. As previously mentioned, the number of measurement points grows quadratically with the complete antenna size. Therefore, a significant level of redundancy is added when calculating the individual contributions of the small subdomains.

As shown in [38], the field radiated by each subdomain can be computed in a non-redundant grid of samples and then interpolated to the desired points. In MSWA, the subdomains' field will be computed evaluating the SWE summation on a coarse grid of points, and then interpolated to the final measurement grid before the aggregation of all subdomain contributions. To obtain significant computational gains, the interpolation process must be performed following a multi-level scheme in which the fields are recursively aggregated.

\section{B. Recursive Aggregation and Interpolation}

Here, we assume a binary domain decomposition in which the AUT enclosing surface is recursively divided into a hierarchy of subdomains with linear sizes reduced by a factor of roughly 2 at each level. To obtain subdomains with half the size on each level, a parent subdomain is divided in four child subdomains, with two divisions on each dimension (Fig. 3). To compute the field radiated by a given subdomain, the fields of 
its corresponding child subdomains are aggregated, except in the highest subdivision level, in which the fields are calculated evaluating the subdomain's SWE. Because on each additional level, the subdomain size is reduced by 2 , so does the number of non-redundant field samples on each angular dimension.

Assuming an AUT whose enclosing surface is divided with $V$ levels, the total number of subdomains in the last level is $2^{V} \times 2^{V}$, so there exists $2^{V} \times 2^{V}$ spherical wave expansions. The aggregation is performed as follows [29]:

1) The process starts at last level $V$, in which the field of each subdomain is computed in a non-redundant angular grid of $p \times p$ points evaluating its SWE. The number of operations of this step is of the order $(p \times p)\left(2^{V} \times 2^{V}\right)=p^{2} 2^{2 V}$.

2) On level $V-1$, the subdomains are grouped in quartets (a pair on each dimension) and their fields are aggregated. Because subdomain size in this level is roughly the double than in the previous one, fields must be interpolated before being aggregated. This interpolation roughly doubles the sampling rate on each dimension and because the number of subdomains has been divided by 4 , the total number of field samples remains approximately constant: $(2 p \times 2 p)\left(\frac{2^{V}}{2} \times \frac{2^{V}}{2}\right)=p^{2} 2^{2 V}$.

3) Step 2 is repeated recursively from level $v=V-1$ to $v=$ 0 until all subdomain contributions are aggregated, and the field radiated by the complete AUT is obtained. On each level the number of samples is multiplied by 4 and the number of subdomains divided by 4 . Thus, the total number of samples is roughly $p^{2} 2^{2 V}$ too.

As a visual example, Fig. 3 depicts an AUT modelled with 16 subdomains using a hierarchy of $V=2$ levels. On level 2 the fields of the 16 subdomains are calculated evaluating the SWEs. A schematic representation of the fields radiated by 4 subdomains is depicted in the lower part of Fig. 3. To reach level 1, the fields of each parent subdomain are calculated combining the fields of its child subdomains. Prior the combination, the four sets of fields are interpolated to double the sampling rate. Though it is not depicted, level 0 will correspond to the total antenna and will be the aggregation of
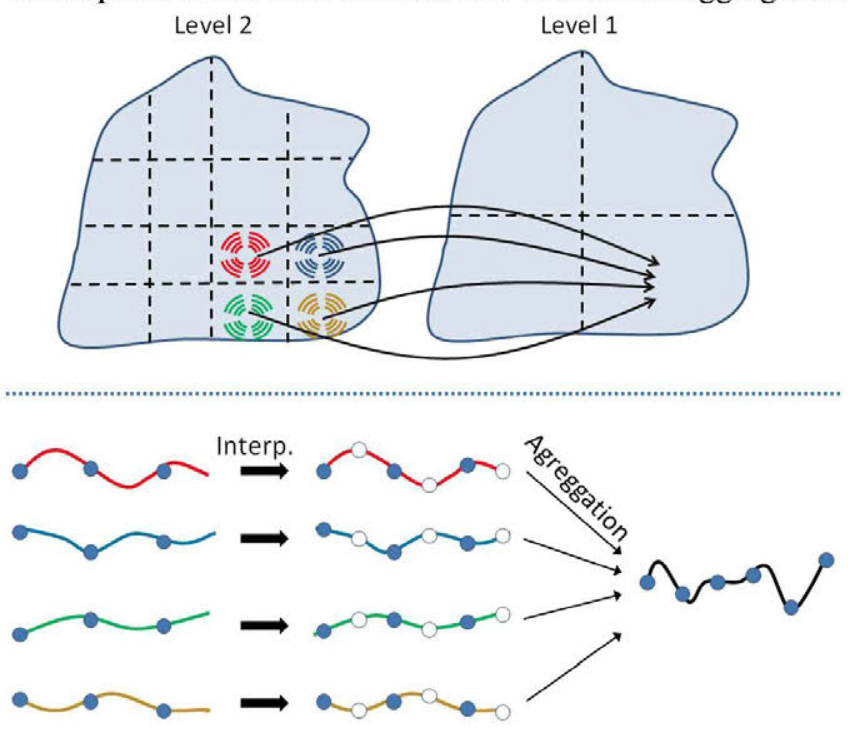

Fig. 3. Recursive domain decomposition and representation of the interpolation-aggregation step of one subdomain. the 4 remaining subdomains.

\section{Computational Complexity Analysis}

If the electrical size of the AUT increases, the number of levels must grow in order to keep a fixed subdomain size on the last level $V$. Therefore, $V$ grows with $O\left(\log _{2}(k a)\right)$ because with each additional level, the size is doubled. On each level, 4 groups of $p^{2} 2^{2 V}$ samples must be interpolated from $p^{2} 2^{2 V}$ points of the previous level and then the samples of all groups must be aggregated. So, if local interpolation is used, on each level the cost is $O\left(p^{2} 2^{2 V}\right)$. Because we have imposed that the subdomain size on the last level must be fixed, $p$ is constant too, so $O\left(p^{2} 2^{2 V}\right)=O\left(2^{2 V}\right)=O\left((k a)^{2}\right)$, and multiplying by the number of levels we obtain the overall computational complexity $O\left((k a)^{2} \log (k a)\right)$. This constitutes a substantial improvement with respect to the $O\left((k a)^{4}\right)$ cost of the conventional matrix product. Therefore, MSWA is suited for electrically large problems thanks to its excellent scalability.

\section{TRANSFORMATION RESULTS}

This section demonstrates the capabilities of the proposed transformation algorithm using as AUT a simulation model of a Yagi-Uda antenna array. The array is made up of 24 by 24 antennas separated $0.8 \lambda$, fed with a uniform amplitude and phase distribution. Each Yagi-Uda is composed of three dipoles separated $1 \lambda$. The antenna used as probe is another Yagi-Uda identical to the array individual element. As such, an antenna measurement scenario is simulated using the AUT and probe analytical models. The output voltage of the probe is calculated for two orthogonal polarizations over a measurement surface given in spherical coordinates:

$$
r=\sqrt{40 \sin ^{2} \theta+20 \cos ^{2} \theta} \lambda
$$

Using (3) the angular step required for the measurement is obtained $\Delta \theta=\Delta \varphi=\frac{\pi}{N}$ but instead of using a classical equiangular sampling, a spiral sampling has been simulated (see Fig. 4). In addition, the simulated near-field is contaminated with a controlled white gaussian noise level.

To perform the near-field to far-field transformation it is necessary to define the MSWE representation. The equivalent surface selected is the combination of the 6 faces of a prism enclosing the AUT. Then, each face is recursively divided in

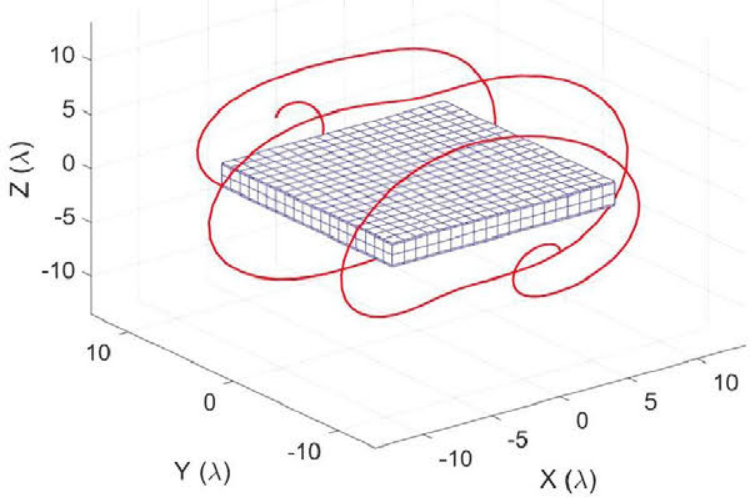

Fig. 4. Subdomain structure enclosing the AUT and simplified representation of the measurement path. 
square subdomains reaching a total of $20 \times 20$ subdomains on the two largest faces. Each subdomain is replaced by an equivalent $\mathrm{SWE}$ of $N=15$. Once the MSWE representation is defined, the iterative inversion algorithm can be started to obtain the expansion coefficients. In the given example, $\mathrm{CG}$ has been applied, stopping the iterations with a noise level residual.

The transformed far-field has been depicted in Fig. 5 for two different levels of SNR along with the analytical solution using the dipole model. Even for a low value of SNR (15 dB), the transformation shows an excellent agreement with the reference. The selected equivalent surface gives information on the AUT's physical size, which can be viewed as a spatial filter that removes the rapid field oscillations of the gaussian noise, leading to such a low transformation error.

\section{A. Interpolation and Scalability Analysis}

Interpolation plays a key role in MSWA as it enables the reduction in computational complexity of the transformation algorithm. When climbing down the hierarchical tree, the child contributions are interpolated and aggregated. All these steps have been listed in the previous sections, but how the fields are sampled and interpolated remains to be defined.

The number of samples to represent the field radiated by a given subdomain over the measurement surface can be kept to the minimum using a given surface parametrization and phase compensating factor as demonstrated in [38]. Based on that formulation, prior interpolation the child fields are compensated by a phase factor that partially cancels the rapid field oscillations [29]. After the interpolation, the phase compensation is removed and then the child fields aggregated.

A uniform sampling in $(\theta, \varphi)$ has been selected and spline, cubic and linear interpolations have been used. MSWA relies on local interpolation schemes to achieve low computational complexity, so global interpolation would degrade the scalability. As a result, some oversampling must be applied to the fields to compensate the non-optimal local interpolation.

In order to assess the interpolation error, the following test is conducted. The MSWA operator is compared with the exact solution of matrix vector product $C Q$ for several sampling rates and interpolation methods. To that end we examine the

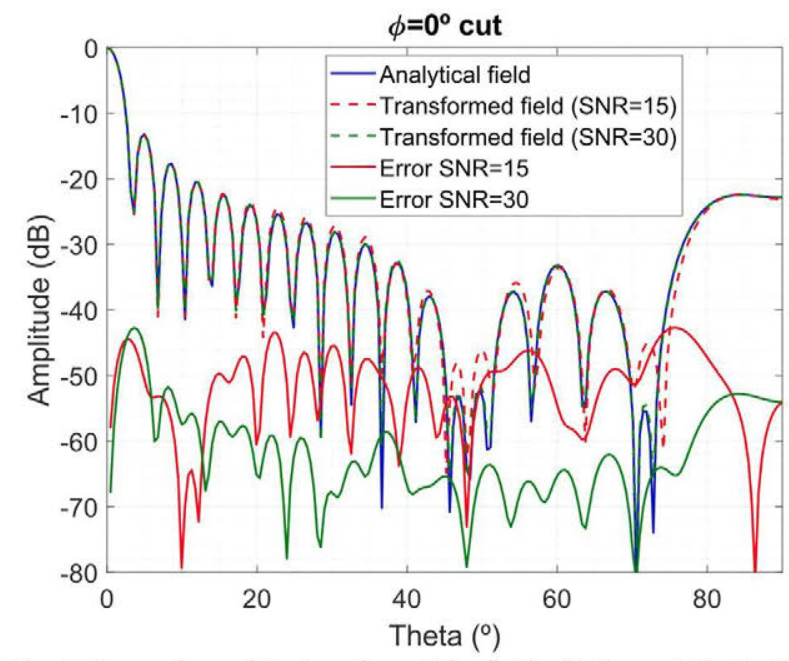

Fig. 5. Comparison of the transformed far-field with the analytical solution. dependence on these parameters of the normalized rms error defined as:

$$
\epsilon_{M S W A}=\sqrt{\frac{\sum_{i=1}^{K}\left|W_{M S W A}[i]-W_{C Q}[i]\right|^{2}}{\sum_{i=1}^{K}\left|W_{C Q}[i]\right|^{2}}}
$$

where $W_{M S W A}$ and $W_{C Q}$ are the measurement vectors calculated with MSWA and matrix product respectively, and $K$ the number of measurement points. MSWA replaces the matrix product in the $\mathrm{CG}$ algorithm so it is important that the approximation error is low to achieve a good level of convergence.

Fig. 6 shows the obtained errors as a function of the sampling rate for linear, cubic and spline interpolators along with the operator computation time. The listed sampling rates are referred to the number of samples in each angular dimension in the smallest subdomain level. Higher order interpolations obtain lower errors at the cost of higher computation times. However, the increase in time is small compared to the obtained error reduction. Therefore, the spline is the best candidate for its integration in MSWA.

Finally, the algorithm's computational complexity is analyzed by gradually increasing the AUT electrical size adding more elements to the array. As the AUT grows, the measurement surface and number of measurement points are increased proportionally as well. The simulation times of one CG iteration using MSWA are shown in Fig. 7. To highlight the scalability improvement, the same test has been performed replacing the MSWA operator by its matrix multiplication counterpart. Only data up to 20 wavelengths were obtained due to memory overflow with the matrix operator.

Simulation times of Fig. 7 have been obtained on a commercial Intel i5@16GB PC in MATLAB. Absolute simulation times are highly dependent on the implementation problem, computer and programming language. The most relevant result is the drastic improvement in computational complexity from $O\left((k a)^{4}\right)$ algorithms [19],[20],[22],[24],[25] to $O\left((k a)^{2} \log (k a)\right)$ which makes it suitable for electrically large problems. A comparison with a traditional spherical far-

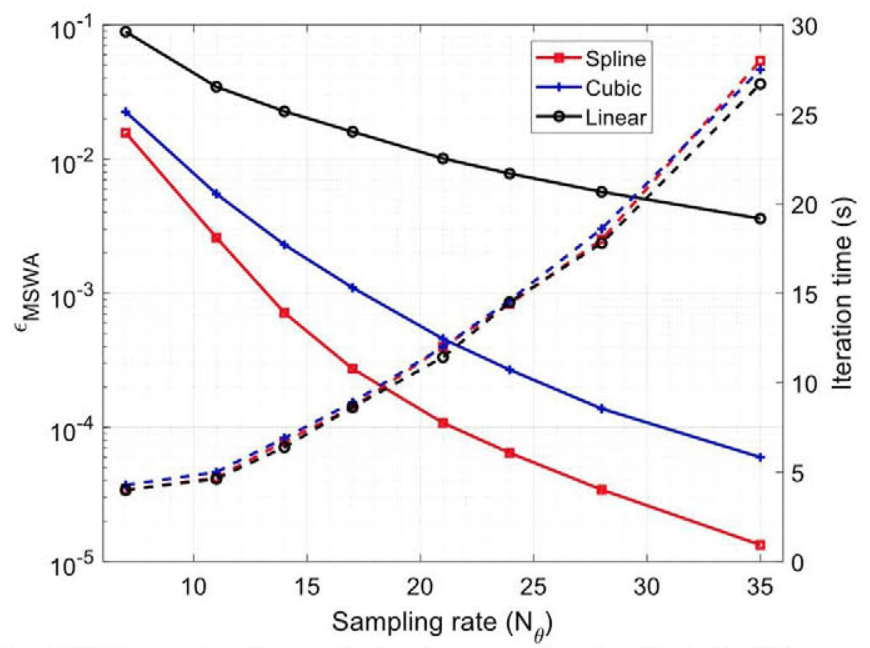

Fig. 6. RMS error (continuous line) and computation time (dashed) of the MSWA operator for several interpolation and sampling schemes. 


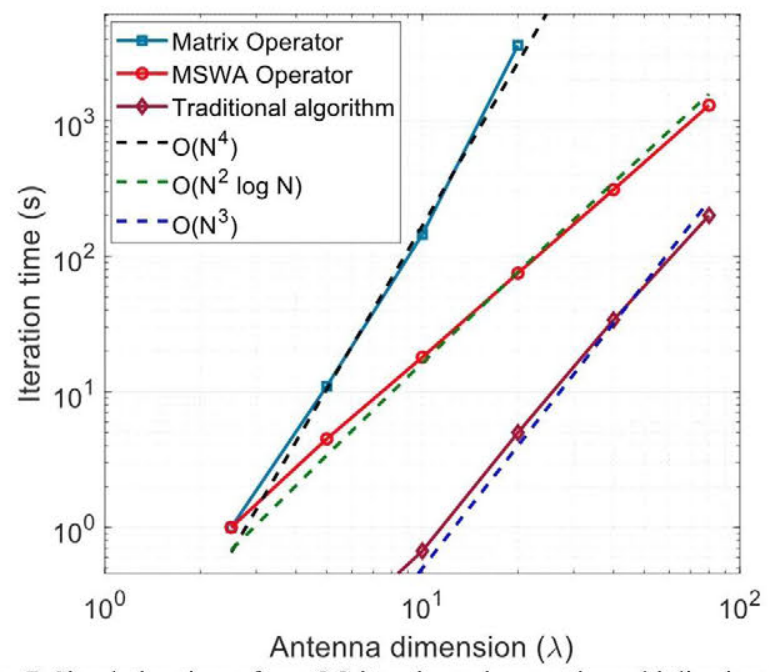

Fig. 7. Simulation time of one CG iteration using matrix multiplication and MWSA operator compared with the traditional canonical transformation.

field transformation [5] implemented in MATLAB is also shown in Fig. 7. Of course, this algorithm is only applicable to spherical surfaces scanned with mono mode probes and equiangular sampling, so a canonical problem of the same electrical dimensions is solved instead to serve as a reference. This traditional algorithm shows the well known $O\left((k a)^{3}\right)$ scalability, which is higher. Despite this, the proposed algorithm cannot compete in absolute simulation times with the traditional technique because it involves more intensive operations. Comparison with [23] could not be made but results similar to [5] are expected, as it is based on the same operations. Therefore, the proposed technique should only be applied for cases where the others [5],[23] are not applicable (arbitrary scanning geometries and/or higher order probes).

Fig. 8 shows the transformation results for the largest simulated Yagi-Uda array corresponding to an electrical dimension of $80 \lambda \times 80 \lambda$. To evaluate the influence of the MSWA interpolation errors, the measurements have been simulated noise-free and with a sampling rate of $N_{\theta}=20$. The interpolation error limits the convergence of the $\mathrm{CG}$ algorithm to a given level which is of the same magnitude than the

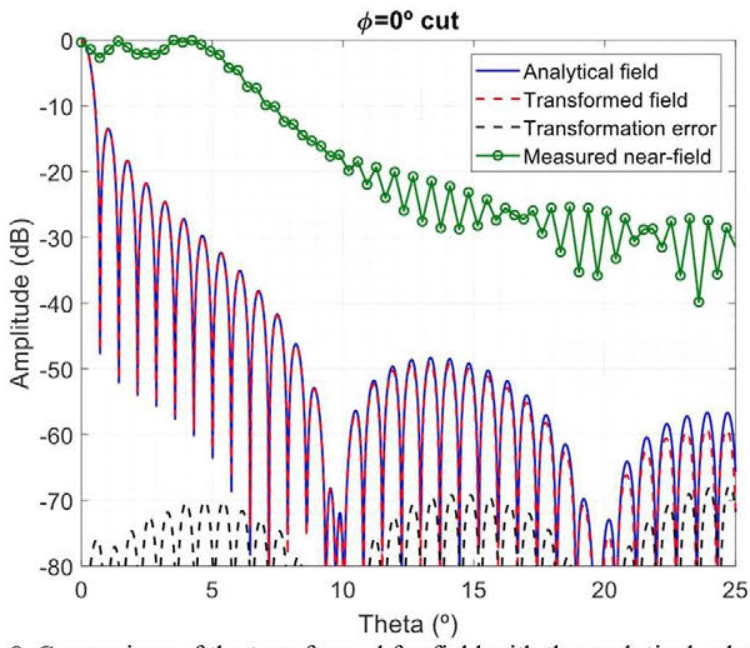

Fig. 8. Comparison of the transformed far-field with the analytical solution for an electrically large antenna using MWSE. observed transformation errors. In this case, the selected sampling rate gives an interpolation error around $10^{-4}$ which roughly corresponds to the obtained transformation errors (around $-80 \mathrm{~dB}$ ). This fact offers a very useful way of adjusting the algorithm depending on the desired accuracy.

\section{B. Measurement Results}

Finally, the proposed algorithm is applied to measured data, to assess its robustness against real noise, drift and other nonideal effects. The measurements have been performed at the anechoic chamber of the Technical University of Madrid (UPM). Although this technique is mainly addressed to the farfield transformation of near-fields measured over arbitrary surfaces, due to the lack of appropriate hardware only classical acquisition surfaces will be presented in this section.

The first test consists in a $2 \times 2$ patch array antenna measured in a spherical near field system using as probe a conical horn at $1 \mathrm{GHz}$. The distance between probe and antenna is $5 \mathrm{~m}$ and an angular sampling of $\Delta \theta=\Delta \varphi=6^{\circ}$ with two orthogonal polarizations is used. The near-field is transformed using as equivalent surface a $2 \lambda \times 2 \lambda \times 2 \lambda$ cube enclosing the AUT. In addition, a far-field transformation using the commercial software SNIFT [39] is performed to be used for reference. Fig. 9 depicts the copolar and crosspolar patterns of a $\varphi=45^{\circ}$ cut. The transformation errors are quite small obtaining an excellent agreement.

For the second example, the VAST12 (VAlidation STandard) antenna is measured in a planar near-field set up at $12 \mathrm{GHz}$. This antenna was used for an intercomparison campaign in the frame of the European Project "Antenna Network of Excellence" and those results are used for comparison [40]. The antenna is an offset reflector of $25 \mathrm{~cm}$ of radius and its near-field is measured in a $1.8 \times 1.8 \mathrm{~m}$ plane at a distance of $80 \mathrm{~cm} .0 .5 \lambda$ sampling rate is selected, and vertical and horizontal polarizations are acquired with a standard gain horn as probe. The defined equivalent surface is a plane located at the AUT aperture. This results in an equivalent problem only valid for the $z>0$ subspace, which is more than enough for this case as the measured near-field only provides information for $\theta<45^{\circ}$ according to the well-known criterion for planar

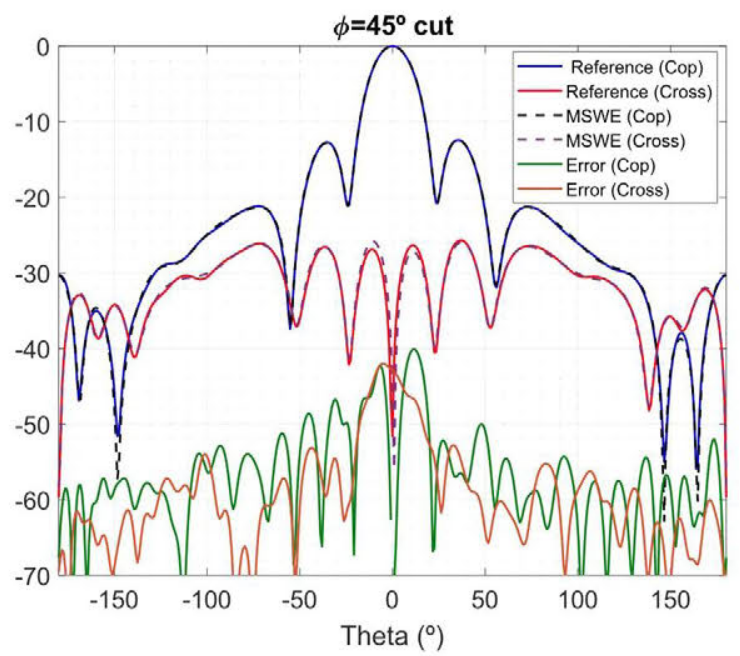

Fig. 9. Patch array co and cross polar transformed far-field patterns. 
measurements[41].

The transformed far-field main cuts are depicted in Fig. 10. For comparison, a standard planar far-field transformation using plane wave spectrum (PWS) has been performed and depicted too, obtaining very similar results down to a level of $50 \mathrm{~dB}$ in the copolar component. The crosspolar component for the vertical cut shows significant differences, but it is comparable to the results obtained by other facilities in the intercomparison campaign. To obtain a more accurate reference, the antenna has been measured in a spherical nearfield system and transformed with SNIFT and displayed along the rest of the curves. PWS and MSWE transformation show similar errors with respect to the reference, which can be attributed to the measurement plane truncation.

\section{Conclusions}

This paper presents an algorithm for the far-field transformation of near-fields measured on arbitrary surfaces. The algorithm is based on replacing the AUT by an equivalent radiating surface which is recursively divided in a multi-level subdomain hierarchy. On the last level, the surface subdomains
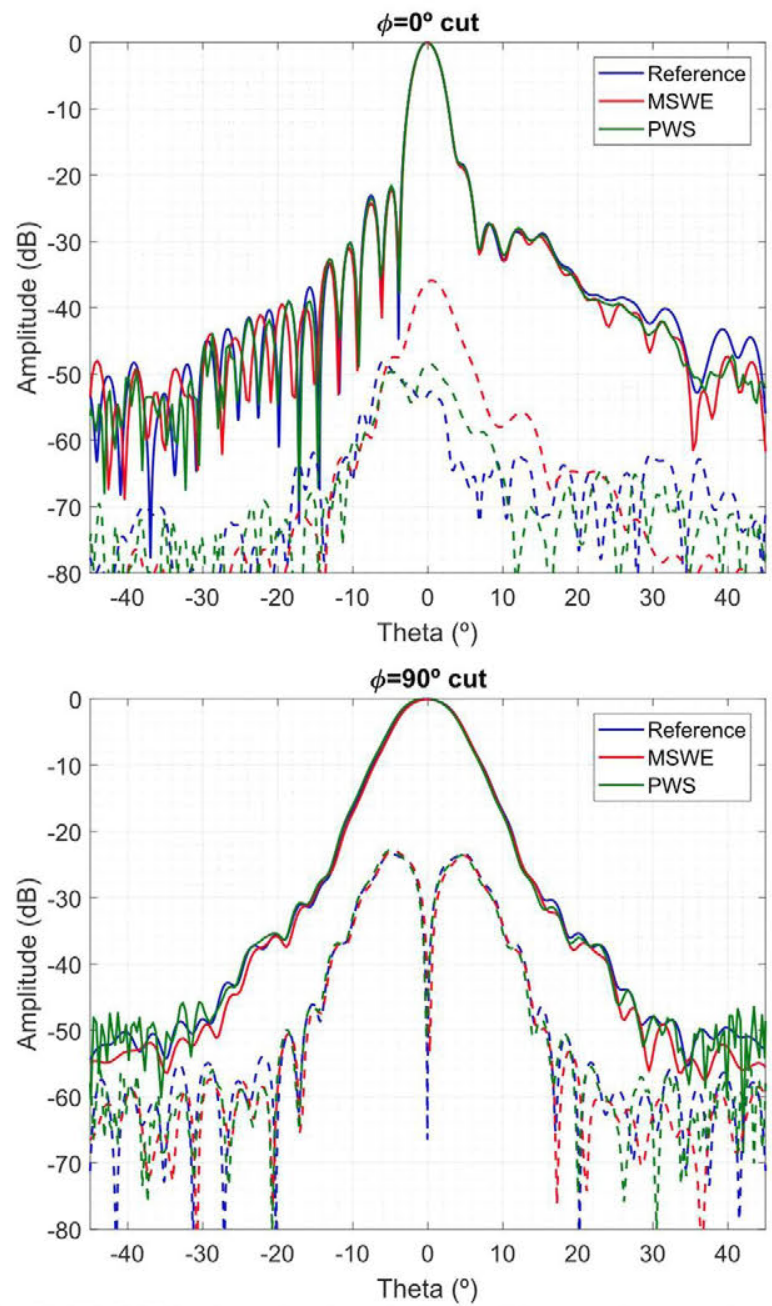

Fig. 10. VAST12 horizontal and vertical cuts of the co and cross polar transformed far-field using SNIFT (reference) from a spherical measurement, and MWSE and PWS from a planar measurement. are modelled with equivalent spherical wave expansions. Then, an iterative inversion algorithm is started to obtain all SWE coefficients. Each iteration requires the calculation of the radiated fields by the expansions, which is performed efficiently following an interpolation-aggregation multilevel scheme. When the expansion coefficients are obtained, these are evaluated at far-field and combined to obtain the AUT radiation pattern. In addition, probe correction is incorporated just by adding its radiation pattern as a spatial weighting function in the first steps. The algorithm has been tested using simulated and measured data, showing low transformation errors, robustness against noise and drastic simulation time savings due to its low computational complexity compared with other explicit matrix inversion algorithms. For the simulated data, non-conventional scanning surfaces have been used to demonstrate the potential capabilities. The use of measured data was only possible with canonical surfaces. Verification of the algorithm with scanned data over arbitrary surfaces remains as a future task, which can be accomplished by means of extra hardware (UAVs, robotic positioners) or the combination of canonical surfaces (cylinder with two caps, hemisphere with planar cap).

\section{REFERENCES}

[1] "IEEE Recommended Practice for Near-Field Antenna Measurements," in IEEE Std 1720-2012, vol., no., pp.1-102, 5 Dec. 2012.

[2] "IEEE Standard Test Procedures for Antennas," in ANSI/IEEE Std 1491979 , vol., no., pp. 0-1, 1979.

[3] T. B. Hansen and A. D. Yaghjian, Plane-Wave Theory of Time Domain Fields. New York: Wiley-IEEE Press, 1999.

[4] W. M. Leach, D. T. Paris, "Probe-Compensated Near-Field Measurements on a Cylinder," in IEEE Transactions on Antennas and Propagation, vol. AP-21, pp. 435-445, July 1973.

[5] J. E. Hansen, "Spherical Near-Field Antenna Measurements," London, U.K.: Peter Peregrinus, 1988.

[6] O. Breinbjerg, "Spherical Near-Field Antenna Measurements-The Most Accurate Antenna Measurement Technique," IEEE International Symposium on Antennas and Propagation, Puerto Rico, pp. 1019-1020, June 2016.

[7] A. Geise et al., "A Crane-Based Portable Antenna Measurement System - System Description and Validation," in IEEE Transactions on Antennas and Propagation, vol. 67, no. 5, pp. 3346-3357, May 2019

[8] M. García-Fernández et al., "Antenna Diagnostics and Characterization Using Unmanned Aerial Vehicles," in IEEE Access, vol. 5, pp. 23563$23575,2017$.

[9] J.A. Gordon, D.R. Novotny, M.H. Francis, R.C. Wittmann, M.L. Butler, A.E. Curtin and J.R. Guerrieri, "Millimeter-Wave Near-Field Measurements Using Coordinated Robotics," in IEEE Transactions on Antennas and Propagation, vol. 63, pp.5351-5362, December 2015.

[10] T. Laitinen, "Double phi-Step theta-Scanning Technique for Spherical Near-Field Antenna Measurements," in IEEE Transactions on Antennas and Propagation, vol. 56, no. 6, pp. 1633-1639, June 2008.

[11] T. Laitinen and O. Breinbjerg, "A First/Third-Order Probe Correction Technique for Spherical Near-Field Antenna Measurements Using Three Probe Orientations," in IEEE Transactions on Antennas and Propagation, vol. 56, no. 5, pp. 1259-1268, May 2008.

[12] T. Laitinen, S. Pivnenko, J. M. Nielsen and O. Breinbjerg, "Theory and Practice of the FFT/Matrix Inversion Technique for Probe-Corrected Spherical Near-Field Antenna Measurements With High-Order Probes," in IEEE Transactions on Antennas and Propagation, vol. 58, no. 8, pp. 2623-2631, Aug. 2010.

[13] T. B. Hansen, "Spherical Near-Field Scanning With Higher-Order Probes," in IEEE Transactions on Antennas and Propagation, vol. 59, no. 11, pp. 4049-4059, Nov. 2011. 
[14] L. J. Foged, F. Saccardi, A. Giacomini, "Probe Correction Technique of Arbitrary Order for High Accuracy Spherical Near Field Antenna Measurements," in AMTA, Austin, Texas, USA, 2016.

[15] F. D'Agostino, F. Ferrara, C. Gennarelli, R. Guerriero and M. Migliozzi, "Fast and Accurate Far-Field Prediction by Using a Reduced Number of Bipolar Measurements," in IEEE Antennas and Wireless Propagation Letters, vol. 16, pp. 2939-2942, 2017.

[16] F. D'Agostino, F. Ferrara, C. Gennarelli, G. Gennarelli, R. Guerriero and M. Migliozzi, "On the Direct Non-Redundant Near-Field-to-Far-Field Transformation in a Cylindrical Scanning Geometry," in IEEE Antennas and Propagation Magazine, vol. 54, no. 1, pp. 130-138, Feb. 2012.

[17] F. D'Agostino, F. Ferrara, C. Gennarelli, R. Guerriero and M. Migliozzi, "Non-Redundant Spherical NF - FF Transformations Using Ellipsoidal Antenna Modeling: Experimental Assessments [Measurements Corner]," in IEEE Antennas and Propagation Magazine, vol. 55, no. 4, pp. 166-175, Aug. 2013.

[18] Cosme Culotta-López, Arya Bangu, Arash Behboodi, Dirk Heberling, Rudolf Mathar, "A Compressed Sampling for Spherical Near-Field Measurements," in AMTA, Williamsburg, Virginia, USA, 2016.

[19] M. Farouq, M. Serhir and D. Picard, "Antenna Far-Field Assessment From Near-Field Measured Over Arbitrary Surfaces," in IEEE Transactions on Antennas and Propagation, vol. 64, no. 12, pp. 51225130, Dec. 2016.

[20] R. Cornelius and D. Heberling, "Spherical Near-Field Scanning With Pointwise Probe Correction," in IEEE Transactions on Antennas and Propagation, vol. 65, no. 2, pp. 995-997, Feb. 2017

[21] L. J. Foged, F. Saccardi, F. Mioc and P. O. Iversen, "Spherical Near Field Offset Measurements using Downsampled Acquisition and Advanced NF/FF Transformation Algorithm," 2016 10th European Conference on Antennas and Propagation (EuCAP), Davos, 2016, pp. 1-3.

[22] R. Cornelius and D. Heberling, "Correction of Non-Ideal Probe Orientations for Spherical Near-Field Antenna Measurements," in AMTA, Atlanta, GA, USA, 2017.

[23] R. C. Wittmann, B. K. Alpert and M. H. Francis, "Near-field, sphericalscanning antenna measurements with nonideal probe locations," in IEEE Transactions on Antennas and Propagation, vol. 52, no. 8, pp. 2184-2187, Aug. 2004

[24] Y. Alvarez, F. Las-Heras and M. R. Pino, "Reconstruction of Equivalent Currents Distribution Over Arbitrary Three-Dimensional Surfaces Based on Integral Equation Algorithms," in IEEE Transactions on Antennas and Propagation, vol. 55, no. 12, pp. 3460-3468, Dec. 2007.

[25] H. Chou, P. H. Pathak, S. Tuan and R. J. Burkholder, "A Novel Far-Field Transformation via Complex Source Beams for Antenna Near-Field Measurements on Arbitrary Surfaces," in IEEE Transactions on Antennas and Propagation, vol. 65, no. 12, pp. 7266-7279, Dec. 2017.

[26] W. C. Chew, J.-M. Jin, E. Michielssen, J. Song, "Fast and Efficient Algorithms in Computational Electromagnetics," MA, Boston:Artech House, 2001.

[27] C. H. Schmidt and T. F. Eibert, "Multilevel Plane Wave Based Near-Field Far-Field Transformation for Electrically Large Antennas in Free-Space or Above Material Halfspace," in IEEE Transactions on Antennas and Propagation, vol. 57, no. 5, pp. 1382-1390, May 2009.

[28] T. F. Eibert, E. Kılıç, C. Lopez, R. A. M. Mauermayer, O. Neitz, G. Schnattinger, "Electromagnetic field transformations for measurements and simulations (invited paper)", Prog. Electromagn. Res., vol. 151, pp. 127-150, 2015

[29] Y. Brick and A. Boag, "Multilevel Nonuniform Grid Algorithm for Acceleration oIntegral Equation-Based Solvers for Acoustic Scattering," in IEEE Transactions on Ultrasonics, Ferroelectrics, and Frequency Control, vol. 57, no. 1, pp. 262-273, Jan. 2010.

[30] A. Boag, "A Fast Physical Optics (FPO) Algorithm for High Frequency Scattering," in IEEE Transactions on Antennas and Propagation, vol. 52, no. 1, pp. 197-204, Jan. 2004.

[31] A. Gergel, Y. Brick and A. Boag, "Fast Antenna Diagnosis Algorithm Using Oblate Spheroidal Nonuniform Grids," in IEEE Transactions on Antennas and Propagation, vol. 64, no. 10, pp. 4197-4207, Oct. 2016.

[32] O. M. Bucci, C. Gennarelli and C. Savarese, "Representation of Electromagnetic Fields over Arbitrary Surfaces by a Finite and Nonredundant Number of Samples," in IEEE Transactions on Antennas and Propagation, vol. 46, no. 3, pp. 351-359, March 1998.
[33] F. Rodriguez Varela, M. Sierra Castañer and B. Galocha Iragüen, "MultiLevel Spherical Wave Expansion for Fast Near-Field to Far-Field Transformation," 2018 AMTA 2018 Proceedings, Williamsburg, VA, 2018, pp. 1-6.

[34] Constantine A. Balanis, "Antenna Theory: Analysis and Design," 3th Edition, Wiley, New Jersey, 2005, pp. 133-144.

[35] M. Serhir, P. Besnier and M. Drissi, "Antenna Modeling Based on a Multiple Spherical Wave Expansion Method: Application to an Antenna Array," in IEEE Transactions on Antennas and Propagation, vol. 58, no. 1, pp. 51-58, Jan. 2010.

[36] Y. Alvarez, F. Las-Heras and M. R. Pino, "Probe-distortion correction for the sources reconstruction method," in IEEE Antennas and Propagation Magazine, vol. 50, no. 6, pp. 117-124, Dec. 2008.

[37] Y. Saad, Iterative Methods for Sparse Linear Systems, 2nd edition, Society for Industrial and Applied Mathematics, 2003.

[38] O. M. Bucci, C. Gennarelli, C. Savarese, "Representation of Electromagnetic Fields Over Arbitrary Surfaces by a Finite and Nonredundant Number of Samples", in IEEE Transactions on Antennas and Propagation, vol. 46, no. 3, pp. 351-359, Mar. 1998.

[39] SNIFT Software, TICRA, Copenhagen, Denmark, www.ticra.com.

[40] S. Pivnenko, J.E. Pallesen, O. Breinbjerg, M.S. Castaner, P.C. Almena, C.M. Portas, J.L: Besada, J. Romeu, S. Blanch, J.M. Gonzalez-Arbesu, C. Sabatier, A. Calderone, G. Portier, H. Eriksson, J. Jackrisson. "Comparison on Antenna Measurement Facilities With the DTU-ESA 12 GHz Validation Standard Antenna Within the EU Antenna Centre of Excelence," in IEEE Transactions on Antennas and Propagation, vol. 57 no. 7, pp. 1863-1878, July 2009.

[41] A. D. Yaghjian, "Upper bound errors in far-field antenna parameters determined from planar near-field measurements, " Nat. Bur. Stand., Boulder, CO, Tech. Rep. NBS Tech. Notes 667, 1975. 